\title{
EDITORIAL
}

\section{M.B.E. IN CANADA}

1965 was a busy year in the Medical and Biological Engineering field, dominated perhaps by the great Conference of interested persons in Tokyo. One important event was the recognition, by the Administrative Council of the International Federation, of the application for affiliation of the Canadian National Group. This group has (or had, at the time of application) 32 members, under the presidency of JoHN A. HopPS, and has shown outstanding support for the Journal in a succession of excellent papers. I have, therefore, great pleasure in announcing the first Canadian National Conference, as follows:

\section{Ist Canadian Medical and Biological \\ Engineering Conference}

To be held at the National Research Council, Ottawa, September 8th and 9th, 1966. Deadline for contributed papers, 21 st May, 1966. 350-word abstracts to be submitted in triplicate by 21 st May to:

R. Cobbold (Chairman, Papers Committee),

Department of Electrical Engineering,

University of Saskatchewan,

Saskatoon, Saskatchewan,

Canada.

Sponsored by: Canadian Society for Medical and Biological Engineering.

\section{CORRIGENDUM}

READERS are asked to note that, at the end of the letter from Mr. B. V. MARKevitch (Med. Electron. \& biol. Engng, 3, 322, 1965) the reference to the patent of Marchal and Marchal did not form part of the text of the original latter submitted by Mr. Markevitch and was not authorized by him. My apologies are due to Mr. Markevitch for this accretion.

P.E.K.D. 